\title{
molecules
}

ISSN 1420-3049

www.mdpi.com/journal/molecules

Article

\section{OxymaPure/DIC: An Efficient Reagent for the Synthesis of a Novel Series of 4-[2-(2-Acetylaminophenyl)-2-oxo-acetylamino] Benzoyl Amino Acid Ester Derivatives}

\author{
Ayman El-Faham ${ }^{1,2, *}$, Zainab Al Marhoon ${ }^{1}$, Ahmed Abdel-Megeed ${ }^{3,4}$ and \\ Fernando Albericio ${ }^{5,6,7,8, *}$
}

1 Department of Chemistry, College of Science, King Saud University, P.O. Box 2455, Riyadh 11451, Saudi Arabia; E-Mail: zanzaki20042000@yahoo.com

2 Department of Chemistry, Faculty of Science, Alexandria University, P.O. Box 426, Ibrahimia, Alexandria 12321, Egypt

3 Department of Botany and Microbiology, College of Science, King Saud University, P.O. Box 2455, Riyadh 11451, Saudi Arabia; E-Mail: aamahmoud@ksu.edu.sa

4 Department of Plant Protection, Faculty of Agriculture, Saba Basha, Alexandria University, Alexandria 12321, Egypt

5 Institute for Research in Biomedicine (IRB), Barcelona Science Park, Baldiri Reixac 10, Barcelona 08028, Spain

6 CIBER-BBN, Networking Centre on Bioengineering, Biomaterials and Nanomedicine, Barcelona Science Park, Baldiri Reixac 10-12, Barcelona 08028, Spain

7 Department of Organic Chemistry, University of Barcelona, Martí i Franqués 1-11, Barcelona 08028, Spain

8 School of Chemistry \& Physics, University of KwaZulu-Natal, Durban 4001, South Africa

* Authors to whom correspondence should be addressed; E-Mails: aymanel_faham@hotmail.com or aelfaham@ksu.edu.sa (A.E.-F.); albericio@irbbarcelona.org (F.A.); Tel.: +96-611-467-3195 (A.E.-F.); Fax: +96-611-467-5992 (A.E.-F.); +34-93-403-7126 (F.A.).

Received: 24 September 2013; in revised form: 15 November 2013 / Accepted: 20 November 2013 / Published: 28 November 2013

\begin{abstract}
OxymaPure (ethyl 2-cyano-2-(hydroxyimino)acetate) was tested as an additive for use in the carbodiimide (DIC) approach for the synthesis of a novel series of $\alpha$-ketoamide derivatives (4-[2-(2-acetylaminophenyl)-2-oxo-acetylamino]benzoyl amino acid ester derivatives). OxymaPure showed clear superiority to HOBt/DIC or carbodiimide alone in terms of purity and yield. The title compounds were synthesized via the ring opening of $\mathrm{N}$-acylisatin. First, $\mathrm{N}$-acetylisatin was reacted with 4 -aminobenzoic acid under
\end{abstract}


conventional heating as well as microwave irradiation to afford 4-(2-(2-acetamidophenyl)2-oxoacetamido)benzoic acid. This $\alpha$-ketoamide was coupled to different amino acid esters using OxymaPure/DIC as a coupling reagent to afford 4-[2-(2-acetylaminophenyl)-2-oxoacetylamino]benzoyl amino acid ester derivatives in excellent yield and purity. The synthesized compounds were characterized using FT-IR, NMR, and elemental analysis.

Keywords: $N$-acetylisatin; 4-aminobenzoic acid; amino acid esters; DIC; OxymaPure; $\alpha$-ketoamide

\section{Introduction}

$\alpha$-Ketoamides are compounds of interest in organic chemistry and are present in many active pharmaceutical compounds [1-6]. Parallel with the application of the $\alpha$-ketoamide moiety in medicinal chemistry, numerous synthetic methods have been described [7-21]. Several authors have demonstrated that the synthesis of the $\alpha$-ketoamide fragment can be achieved by ring opening of $\mathrm{N}$-acetylisatin (1) by the attack of an amine at C2-carbonyl group of $N$-acetylisatin (Scheme 1) [22-27]. Recently, Cheah et al. [28,29] reported the reaction of $N$-acetylisatin with L- $\alpha$-amino acid esters as a novel class of $N$-glyoxylamide peptide mimics.

Scheme 1. General mechanism for the reaction of $N$-acylisatin (1) with amines.

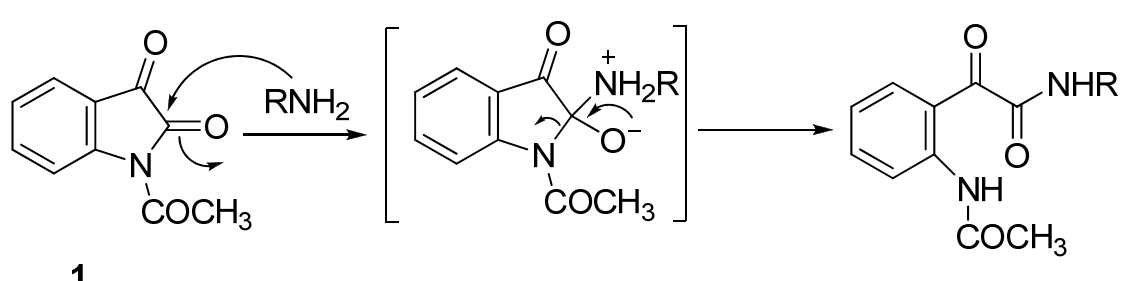

1

Here we present the use of OxymaPure/DIC as a coupling reagent for the synthesis of a novel class of $\alpha$-ketoamide derivatives (4-[2-(2-acetylaminophenyl)-2-oxo-acetylamino]benzoyl amino acid esters).

\section{Results and Discussion}

1-Acetylindoline-2,3-dione ( $N$-acetylisatin, 1) was initially prepared by reaction of isatin with acetic anhydride, using conventional heating under the same conditions as those described in the literature [30,31]. However, we demonstrate that the use of a microwave irradiation, using a multimode reactor (Synthos 3000, Anton Paar GmbH, Graz, Austria, 1,400 W maximum magnetron, method B; Experimental section), renders 1 from isatin and acetic anhydride (Scheme 2) in excellent yield in less reaction time and higher purity than the conventional method, as observed from spectral data. This observation is consistent with data in the literature [30].

$N$-Acetylisatin (1) was then reacted with the poor nucleophile, 4-aminobenzoic acid (2) using conventional heating for $1 \mathrm{~h}$ in methanol as a solvent to afford the product 3 (Scheme 2). The IR and NMR spectral analysis of the product revealed that the reaction proceeded through the ring opening to 
afford the $\alpha$-ketoamide derivative 3 and not the Schiff base derivative, in contrast to what was reported in the reaction of bromo- $N$-acetylisatin with aminobenzoic acid. [32,33] The IR spectra of $\mathbf{3}$ showed three characteristic peaks at 3270,1679 , and $1601 \mathrm{~cm}^{-1}$, corresponding to the $\mathrm{COOH}$, the $\alpha$-ketoamide (COCONH), and $\mathrm{NHCOCH}_{3}$, respectively. The ${ }^{1} \mathrm{H}-\mathrm{NMR}$ of 3 agreed well with the structure, showing eight distinct resonance peaks located at $\delta 1.99\left(\mathrm{~s}, 3 \mathrm{H}, \mathrm{COCH}_{3}\right), 7.27-7.730$ (m, 2H, Ar), 7.62-7.68 (m, 2H, Ar), 7.90 (d, 2H, Ar), 7.90 (d, 2H, Ar), 10.55 (s, 1H, NH), 10.99 $(\mathrm{s}, 1 \mathrm{H}, \mathrm{NH})$, and 13.00 (brs, $1 \mathrm{H}, \mathrm{COOH})$. These peaks were assigned to the acetyl group, aromatic proton (isatin), aromatic proton (4-aminobenzoic acid), two $\mathrm{NHs}$, and $\mathrm{COOH}$ respectively. The ${ }^{13} \mathrm{C}-\mathrm{NMR}$ of 3 also confirmed the structure, showing the characteristic signals at $\delta 161.0,166.1,168.2$, and 188.1 corresponding to the two amide groups, one carbonyl of the carboxyl group, and the $\alpha$-ketoamide group respectively, along with the rest of the expected carbon signals of the compound.

Scheme 2. Synthesis and reaction of $N$-acylisatin (1) with 4-aminobenzoic acid (2).<smiles>O=C1Nc2ccccc2C1=O</smiles><smiles>CC(C)=O</smiles><smiles>CC(=O)N1C(=O)C(=O)c2ccccc21</smiles><smiles>CC(=O)Nc1ccccc1C(=O)C(=O)Nc1ccc(C(=O)O)cc1</smiles>

The same reaction was repeated under microwave irradiation using a multimode reactor (Anton Paar GmbH Synthos 3000, 1,400 W maximum magnetron, method B in the Experimental section) to afford product 3 (Scheme 2) in less reaction time and high purity as shown by its spectral data. The IR and NMR spectra of $\mathbf{3}$ proved its structure and were in agreement with the product obtained by conventional heating.

Recently, OxymaPure (ethyl 2-cyano-2-(hydroxyimino)acetate, Scheme 3) was used as an additive for peptide synthesis in combination with carbodiimides [34]. It displayed an appropriate balance of availability and ease of handling. In addition it was safer than HOBt and showed clear superiority in terms of coupling efficiency [34-42].

Here we tested OxymaPure as an additive for the coupling of compound $\mathbf{3}$ to $\mathrm{H}$-Ala-OMe $\cdot \mathrm{HCl}$. In a general experiment, 3 was preactivated with OxymaPure/DIC for $5 \mathrm{~min}$ in DMF to generate the corresponding active ester (Scheme 3), which reacted directly with $\mathrm{H}$-Ala-OMe$\cdot \mathrm{HCl}$ in the presence of 1 equiv. of DIEA at $0{ }^{\circ} \mathrm{C}$ for $1 \mathrm{~h}$ and then at r.t overnight. After workup and removal of the solvent, product 4 a was obtained as a white solid in $88 \%$ yield (Scheme 3 ).

The reaction of 3 with $\mathrm{H}-\mathrm{Ala}-\mathrm{OMe}$ was then repeated using various coupling reagents (Table 1). The best results were obtained using OxymaPure/DIC; OxymaPure showed clear superiority to HOBt/DIC and carbodiimide alone in terms of yield and purity. DCC consistently showed some impurities from dicyclohexylurea (DCU) as observed from NMR spectra. 
Scheme 3. Synthesis 4-[2-(2-acetylaminophenyl)-2-oxo-acetylamino]benzoyl amino acid ester derivatives $\mathbf{4 a}-\mathbf{i}$.<smiles>CCOC(=O)/C(C#N)=N/OC1CCCCC1</smiles>

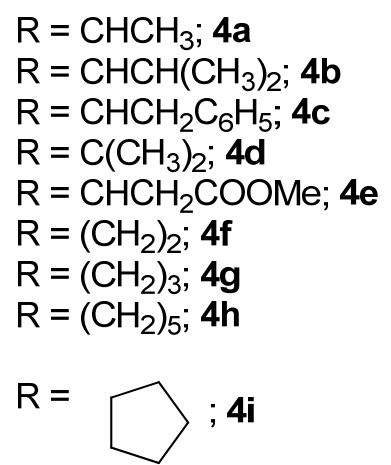<smiles>CCOOC(=O)C(C#N)=NOC(=O)c1ccc(NC(=O)C(=O)c2ccccc2NC(=O)OCc2ccccc2)cc1</smiles>

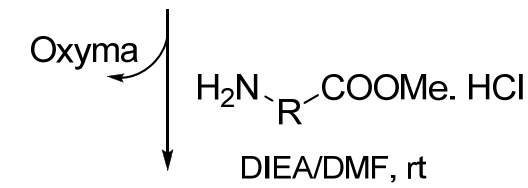<smiles>CC(=O)NC(=O)c1ccc(NC(=O)C(=O)c2ccccc2NC(C)=O)cc1</smiles>

4a-i

Table 1. Reaction of $\mathbf{3}$ with $\mathrm{H}-\mathrm{Ala}-\mathrm{OMe} . \mathrm{HCl}$ using various coupling conditions.

\begin{tabular}{ccc}
\hline Coupling Condition & Yield (\%) & Mp $\left({ }^{\circ} \mathbf{C}\right)$ \\
\hline DIC/Oxyma & 88 & $174-176$ \\
DCC/Oxyma * & 82 & $168-172$ \\
DIC/HOBt & 72 & $172-175$ \\
DCC/HOBt * & 70 & $170-174$ \\
DIC & 60 & $170-173$ \\
DCC * & 60 & $168-173$ \\
\hline
\end{tabular}

* NMR showed impurities corresponding to the dicyclohexylurea byproduct.

The IR spectrum of $4 \mathbf{a}$ showed four characteristic peaks at 3288, 1741, 1672, and $1607 \mathrm{~cm}^{-1}$, corresponding to the $\mathrm{NH}, \mathrm{CO}$-ester, $\alpha$-ketoamide and CO-amide, respectively. The ${ }^{1} \mathrm{H}-\mathrm{NMR}$ showed a doublet peak at $\delta 1.41$, which was related to the $\mathrm{CH}_{3}$ for the alanine unit, two singlet peaks at $\delta 2.00$ and 3.65 for the acetyl and the methyl ester, and three singlet peaks at $\delta 8.73,10.55,10.92$ for three NHs, respectively. The ${ }^{13} \mathrm{C}-\mathrm{NMR}$ also confirmed the structure of $\mathbf{4 a}$, showing signals at $\delta 17.3$ $\left(\mathrm{CHCH}_{3}\right), 24.2\left(\mathrm{NCOCH}_{3}\right), 49.9\left(\mathrm{COOCH}_{3}\right), 52.1(\mathrm{CH}-\mathrm{NH}), 162.3,166.2$, and 169.5 (for three CONHs), $173.8\left(\mathrm{COOCH}_{3}\right)$, and $190.0(\mathrm{COCO})$, along with the remaining carbon residues related to $4 \mathbf{a}$.

Several amino acid esters were prepared following the reported method $[43,44]$ and coupled with 3 using OxymaPure/DIC under the same conditions used for the coupling of H-Ala-OMe. This approach afforded products $\mathbf{4 a - i}$ in excellent yield and purity (Scheme 3, Table 2). The structures of all the compounds synthesized were confirmed by IR, NMR $\left({ }^{1} \mathrm{H}-\mathrm{NMR}\right.$ and $\left.{ }^{13} \mathrm{C}-\mathrm{NMR}\right)$ and elemental analysis. 
Table 2. Yield (\%), $\mathrm{Mp}\left({ }^{\circ} \mathrm{C}\right)$, and Elemental analysis of $\mathbf{4 a}-\mathbf{i}$.

\begin{tabular}{cccccc}
\hline \multirow{2}{*}{ Compd. No } & \multirow{2}{*}{ Yield (\%) } & \multirow{2}{*}{$\mathbf{M p}\left({ }^{\circ} \mathbf{C}\right)$} & \multicolumn{3}{c}{ Elemental Analysis Calcd. (Found) } \\
\cline { 4 - 6 } & & & $\mathbf{C}$ & $\mathbf{H}$ & $\mathbf{N}$ \\
\hline $\mathbf{4 a}$ & 88 & $174-176$ & $61.31(61.60)$ & $5.14(5.34)$ & $10.21(10.00)$ \\
$\mathbf{4 b}$ & 81 & $178-180$ & $62.86(63.02)$ & $5.73(5.96)$ & $9.56(9.581)$ \\
$\mathbf{4 c}$ & 83 & $168-170$ & $66.52(66.67)$ & $5.17(5.24)$ & $8.62(8.88)$ \\
$\mathbf{4 d}$ & 76 & $216-218$ & $62.11(62.38)$ & $5.45(5.66)$ & $9.88(10.07)$ \\
$\mathbf{4 e}$ & 82 & $154-156$ & $66.52(66.33)$ & $5.17(5.26)$ & $8.62(8.90)$ \\
$\mathbf{4 f}$ & 88 & $154-156$ & $61.31(61.09)$ & $5.14(5.23)$ & $10.21(10.48)$ \\
$\mathbf{4 g}$ & 86 & $118-120$ & $62.11(62.37)$ & $5.45(5.67)$ & $9.88(10.04)$ \\
$\mathbf{4 h}$ & 83 & $180-182$ & $63.56(63.38)$ & $6.00(6.13)$ & $9.27(9.53)$ \\
$\mathbf{4 i}$ & 78 & $238-240$ & $63.85(64.06)$ & $5.58(5.65)$ & $9.31(9.04)$ \\
\hline
\end{tabular}

\section{Experimental}

\subsection{General}

The solvents used were of HPLC reagent grade. Melting points were determined with a Mel-Temp apparatus and are uncorrected. Fourier transform infrared spectroscopy (FTIR) spectra was recorded on Nicolet 560 spectrometer. Nuclear magnetic resonance spectra $\left({ }^{1} \mathrm{H}-\mathrm{NMR}\right.$ and ${ }^{13} \mathrm{C}-\mathrm{NMR}$ spectra) were recorded on a JOEL $400 \mathrm{MHz}$ spectrometer with chemical shift values reported in $\delta$ units (ppm) relative to an internal standard. The microwave irradiation used a multimode reactor (Synthos 3000, Anton Paar $\mathrm{GmbH}$, and 1,400 W maximum magnetron). Elemental analyses were performed on Perkin-Elmer 2400 elemental analyzer, and the values found were within $\pm 0.3 \%$ of the theoretical values. Follow-up of the reactions and checks of the purity of the compounds were done by TLC on silica gel-protected aluminum sheets (Type 60 GF254, Merck) and the spots were detected by exposure to a UV-lamp at $\lambda 254 \mathrm{~nm}$ for a few seconds. The compounds were named using ChemDraw Ultra version 11, Cambridge Soft Corporation (Cambridge, MA, USA).

\subsection{Synthesis of 1-Acetyl-1H-indole-2,3-dione (N-acetylisatin) (1)}

Conventional method (A): A mixture of isatin $(0.01 \mathrm{M})$ and acetic anhydride $(5 \mathrm{~mL})$ was refluxed for $5 \mathrm{~h}$. After cooling to r. t., it was left to stand overnight. The precipitate was collected, washed with $96 \%$ ethanol and air-dried.

Microwave method (B): A multimode reactor (Anton Paar GmbH Synthos 3000, 1,400 W maximum magnetron) was used. The initial step was conducted with a 2-Teflon vessels rotor (MF 100). Isatin $(5 \mathrm{mmol})$ was suspended in acetic anhydride $(10 \mathrm{~mL})$ and the reaction was processed by heating the vessels for $5 \mathrm{~min}$. at $80{ }^{\circ} \mathrm{C}$ and holding it at the same temperature for $5 \mathrm{~min}$ (under $0.2 / \mathrm{s}$ bar pressure, $400 \mathrm{~W}$ ). Cooling was accomplished by a fan (for $5 \mathrm{~min}$ ) and the desired product was obtained as a yellow needle in excellent yield without further recrystallization. The spectral data were in accordance with the data reported in the literature [30].

1-Acetyl-1H-indole-2,3-dione (N-acetylisatin) (1) The product was obtained as green crystals, mp: 146-148 ${ }^{\circ} \mathrm{C}$; yields: 79\% (method A); 95\% (method B) (lit. [23] $141{ }^{\circ} \mathrm{C}$, yield 97\%; lit. [30] 137-139 ${ }^{\circ} \mathrm{C}$, 
yield 70\%). ${ }^{1} \mathrm{H}-\mathrm{NMR}\left(\mathrm{CDCl}_{3}\right) \delta(\mathrm{ppm}): 2.69\left(\mathrm{~s}, 3 \mathrm{H}, \mathrm{COCH}_{3}\right), 7.31(\mathrm{t}, J=8.04 \mathrm{~Hz}, 1 \mathrm{H}), 7.69(\mathrm{t}$, $J=7.32 \mathrm{~Hz}, 1 \mathrm{H}), 7.73(\mathrm{~d}, J=7.32 \mathrm{~Hz}, 1 \mathrm{H}), 8.36(\mathrm{~d}, J=8.08 \mathrm{~Hz}, 1 \mathrm{H}) ;{ }^{13} \mathrm{C}-\mathrm{NMR}\left(\mathrm{CDCl}_{3}\right) \delta(\mathrm{ppm}): 26.5$ $\left(\mathrm{COCH}_{3}\right), 118.3,119.2,125.3,126.2,139.0,148.6,158.0$ (CO), 169.8 (CO), 180.2 (CO).

\subsection{Synthesis of 4-(2-(2-Acetamidophenyl)-2-oxoacetamido)benzoic Acid (3)}

Conventional method (A): A mixture of $N$-Acetyl isatin $(0.01 \mathrm{M})$ and 4-amino-benzoic acid $(0.01 \mathrm{M})$ in absolute methanol $(20 \mathrm{~mL})$ was refluxed for $1 \mathrm{~h}$ in the presence of 2-3 drops of glacial acetic acid. After cooling, was filtered and recrystallized from ethanol to afford the product in $96 \%$ yield.

Microwave method (B): A multimode reactor (Synthos 3000 Aton Paar, GmbH, $1400 \mathrm{~W}$ maximum magnetron) was used. The initial step was conducted with 2-Teflon vessels rotor (MF 100) that allows the reaction to be processed under the same conditions. $N$-acetylisatin and 4 -aminobenzoic acid were mixed in methanol as a solvent in the presence or absence of glacial acetic acid (2-3 drops). The individual vessels were placed in the corresponding rotor, and finally the rotor was closed with a protective hood. The vessels were heated for $2 \mathrm{~min}$. at $80{ }^{\circ} \mathrm{C}$ and held at the same temperature for another $2 \mathrm{~min}$ ( $\sim 2$ bar pressure, $400 \mathrm{~W})$. Cooling was accomplished by a fan $(5 \mathrm{~min})$. The final product was washed with cold methanol, and then dried under vacuum to afford the product in a pure state as observed from spectral analysis.

The product was obtained as a pale yellow powder, mp: $238-240{ }^{\circ} \mathrm{C}$; yield $(57 \%$ method A); $(86 \%$ method B). ${ }^{1} \mathrm{H}-\mathrm{NMR}$ (DMSO- $\left.d_{6}\right) \delta$ (ppm): 1.99 (s, 3H, $\mathrm{COCH}_{3}$ ), 7.25-7.30 (m, 2H), 7.62-7.68 $(\mathrm{m}, 2 \mathrm{H}), 7.90(\mathrm{~d}, J=8.79 \mathrm{~Hz}, 2 \mathrm{H}), 7.95$ (d, $J=8.43 \mathrm{~Hz}, 2 \mathrm{H}), 10.55(\mathrm{~s}, 1 \mathrm{H}, \mathrm{NH}), 10.99(\mathrm{~s}, 1 \mathrm{H}, \mathrm{NH})$, 13.00 (brs, 1H, COOH); ${ }^{13} \mathrm{C}-\mathrm{NMR}$ (DMSO-d6) $\delta$ (ppm): $22.8\left(\mathrm{NHCOCH}_{3}\right), 118.7,121.0,123.1,124.3$, 125.4, 129.6, 130.3, 132.9, 136.8, 141.2, $161.00(\mathrm{CONH}), 166.1(\mathrm{CONH}), 168.2(\mathrm{COOH}), 188.1$ (COCO). IR $\left(\mathrm{cm}^{-1}\right): 3270(\mathrm{COOH}), 1679,1601(\mathrm{C}=\mathrm{O})$. Anal. Calcd. for $\mathrm{C}_{17} \mathrm{H}_{14} \mathrm{~N}_{2} \mathrm{O}_{5}: \mathrm{C}, 62.57 ; \mathrm{H}$, 4.32; N, 8.59. Found: C, 62.80; H, 4.21; N, 8.33.

\subsection{General Method for the Synthesis of Amino Acid Esters}

Thionyl chloride $(10 \mathrm{~mL})$ was slowly added to a cold suspension solution of the appropriate amino acid $(50 \mathrm{mmol})$ in methanol $(50 \mathrm{~mL})$ at $0{ }^{\circ} \mathrm{C}$. The reaction mixture was stirred for $8-10 \mathrm{~h}$ and then concentrated on a rotary evaporator. The white precipitate formed was washed with anhydrous ether and then dried under vacuum. All data agreed with the reported data $[43,44]$.

Methyl 1-aminocyclopentane carboxylate $\mathrm{HCl}$. White powder, mp: $204-206{ }^{\circ} \mathrm{C}$, yield $87 \%$. ${ }^{1} \mathrm{H}-\mathrm{NMR}$ (DMSO- $\left.d_{6}\right) \delta(\mathrm{ppm}): 1.62-1.72\left(\mathrm{~m}, 2 \mathrm{H}, \mathrm{CH}_{2} \mathrm{CH}_{2} \mathrm{CH}_{2}\right), 1.81-1.91\left(\mathrm{~m}, 2 \mathrm{H}, \mathrm{HNCH}_{2} \mathrm{CH}_{2} \mathrm{CH}_{2}\right), 1.94-1.96$ (m, $2 \mathrm{H}, \mathrm{CH}_{2} \mathrm{CH}_{2} \mathrm{CO}$ ), 2.07-2.14 (m, 2H, $\mathrm{CH}_{2} \mathrm{CH}_{2} \mathrm{NH}$ ), 3.73 (s, 3H, $\left.\mathrm{COOCH}_{3}\right), 8.81$ (brs, $2 \mathrm{H}, \mathrm{NH}_{2}$ ). ${ }^{13} \mathrm{C}-\mathrm{NMR}\left(\mathrm{DMSO}-d_{6}\right) \delta$ (ppm): 24.3, 35.2, $52.4\left(\mathrm{COOCH}_{3}\right), 63.4,171.8\left(\mathrm{COOCH}_{3}\right)$.

Methyl 3-aminopropionate $\mathrm{HCl}$. White powder, mp: 90-92 ${ }^{\circ} \mathrm{C}$, yield $85 \% .{ }^{1} \mathrm{H}-\mathrm{NMR}\left(\mathrm{DMSO}-d_{6}\right) \delta(\mathrm{ppm})$ : $2.72\left(\mathrm{t}, J=7.35 \mathrm{~Hz}, 2 \mathrm{H}, \mathrm{NH}_{2} \mathrm{CH}_{2} \mathrm{CH}_{2} \mathrm{CO}\right.$ ), 2.98 (m, 2H, $\mathrm{NH}_{2} \mathrm{CH}_{2} \mathrm{CH}_{2} \mathrm{CO}$ ), 3.62 (s, 3H, $\mathrm{COOCH}_{3}$ ), 8.22 (brs, $2 \mathrm{H}, \mathrm{NH}_{2}$ ). 
Methyl 4-aminobutanoate $\mathrm{HCl}$. White powder, mp: $104-106{ }^{\circ} \mathrm{C}$, yield $87 \%$. ${ }^{1} \mathrm{H}-\mathrm{NMR}$ (DMSO- $d_{6}$ ) $\delta$ (ppm): $1.80\left(\mathrm{~m}, 2 \mathrm{H}, \mathrm{NH}_{2} \mathrm{CH}_{2} \mathrm{CH}_{2} \mathrm{CH}_{2} \mathrm{CO}\right), 2.43$ (t, $\left.J=7.0 \mathrm{~Hz}, 2 \mathrm{H}, \mathrm{NH}_{2} \mathrm{CH}_{2} \mathrm{CH}_{2} \mathrm{CH}_{2} \mathrm{CO}\right), 2.78$ (m, $2 \mathrm{H}, \mathrm{NH}_{2} \mathrm{CH}_{2} \mathrm{CH}_{2} \mathrm{CH}_{2} \mathrm{CO}$ ), 3.59 (s, $3 \mathrm{H}, \mathrm{COOCH}_{3}$ ), 8.16 (brs, $2 \mathrm{H}, \mathrm{NH}_{2}$ ).

Methyl 6-aminohexanoate $\mathrm{HCl}$. White powder, $\mathrm{mp}: \quad 104-106{ }^{\circ} \mathrm{C}$, yield $90 \%$. ${ }^{1} \mathrm{H}-\mathrm{NMR}$ $\left(\mathrm{DMSO}-d_{6}\right) \quad \delta$ (ppm): $1.26-1.31 \quad\left(\mathrm{~m}, \quad 2 \mathrm{H}, \quad \mathrm{NH}_{2} \mathrm{CH}_{2} \mathrm{CH}_{2} \mathrm{CH}_{2} \mathrm{CH}_{2} \mathrm{CH}_{2} \mathrm{CO}\right), \quad 1.49-1.57 \quad$ (m, 4H, $\mathrm{NH}_{2} \mathrm{CH}_{2} \mathrm{CH}_{2} \mathrm{CH}_{2} \mathrm{CH}_{2} \mathrm{CH}_{2} \mathrm{CO}$ ), 2.29 (t, $J=7.30 \mathrm{~Hz}, 2 \mathrm{H}, \mathrm{NH}_{2} \mathrm{CH}_{2} \mathrm{CH}_{2} \mathrm{CH}_{2} \mathrm{CH}_{2} \mathrm{CH}_{2} \mathrm{CO}$ ), 2.71 (m, 2H, $\mathrm{NH}_{2} \mathrm{CH}_{2} \mathrm{CH}_{2} \mathrm{CH}_{2} \mathrm{CH}_{2} \mathrm{CH}_{2} \mathrm{CO}$ ), 3.57 (s, $3 \mathrm{H}, \mathrm{COOCH}_{3}$ ), 8.08 (brs, $2 \mathrm{H}, \mathrm{NH}_{2}$ ).

Dimethyl 2-aminosuccinate $\mathrm{HCl}$. White powder, mp: $110-112{ }^{\circ} \mathrm{C}$, yield $82 \% .{ }^{1} \mathrm{H}-\mathrm{NMR}$ (DMSO- $d_{6}$ ) $\delta$ (ppm): 3.03 (q, $\left.J=4.0 \mathrm{~Hz}, 2 \mathrm{H}, \mathrm{COCH}_{2} \mathrm{CHNH}_{2}\right), 3.64$ (s, 3H, $\left.\mathrm{COOCH}_{3}\right), 3.72\left(\mathrm{~s}, 3 \mathrm{H}, \mathrm{COOCH}_{3}\right)$, $4.33\left(\mathrm{~m}, 1 \mathrm{H}, \mathrm{CH}_{2} \mathrm{CHNH}_{2}\right), 8.75$ (brs, $2 \mathrm{H}, \mathrm{NH}_{2}$ ).

\subsection{General Procedure for the Synthesis of $\mathbf{4 a}-\mathbf{i}$}

Acid 3 (1 mmol), Oxyma (1 mmol), and DIC (1 mmol) were mixed in DMF $(5 \mathrm{~mL})$ at $0{ }^{\circ} \mathrm{C}$. The reaction mixture was stirred for $5 \mathrm{~min}$ at $0{ }^{\circ} \mathrm{C}$ to preactivate the acid and generate the active ester, and then DIEA $(1 \mathrm{mmol})$ followed by amino acid ester $(1 \mathrm{mmol})$ were added. The reaction mixture was stirred at $0{ }^{\circ} \mathrm{C}$ for $1 \mathrm{~h}$ and at room temperature overnight. The mixture was then diluted with ethyl acetate $(50 \mathrm{~mL})$ and then extracted with $1 \mathrm{~N} \mathrm{HCl}(2 \times 10 \mathrm{~mL}), 10 \% \mathrm{NaHCO}_{3}(2 \times 10 \mathrm{~mL})$, and saturated $\mathrm{NaCl}(2 \times 10 \mathrm{~mL})$. The organic phase was dried over anhydrous $\mathrm{MgSO}_{4}$, filtered, and the solvent was removed under vacuum. The residue was recrystallized from dichloromethane-hexane to afford the pure product.

Methyl 2-(4-(2-(2-acetamidophenyl)-2-oxoacetamido)benzamido)propanoate (4a). White powder, mp: 174-176 ${ }^{\circ} \mathrm{C}$, yield 88\%. ${ }^{1} \mathrm{H}-\mathrm{NMR}$ (DMSO- $\left.d_{6}\right) \delta(\mathrm{ppm}): 1.41\left(\mathrm{~d}, J=7.32 \mathrm{~Hz}, 3 \mathrm{H}, \mathrm{CHCH}_{3}\right), 2.00(\mathrm{~s}, 3 \mathrm{H}$, $\left.\mathrm{COCH}_{3}\right), 3.65\left(\mathrm{~s}, 3 \mathrm{H}, \mathrm{COOCH}_{3}\right), 4.48\left(\mathrm{~m}, 1 \mathrm{H}, \mathrm{NHCHCH}_{3}\right), 7.45(\mathrm{t}, J=7.2 \mathrm{~Hz}, 1 \mathrm{H}), 7.64(\mathrm{~d}, J=4.4 \mathrm{~Hz}$, $1 \mathrm{H}), 7.68(\mathrm{~d}, J=7.36 \mathrm{~Hz}, 2 \mathrm{H}), 7.89(\mathrm{~m}, 4 \mathrm{H}, \mathrm{Ar}), 8.73(\mathrm{~s}, 1 \mathrm{H}, \mathrm{NH}), 10.55(\mathrm{~s}, 1 \mathrm{H}, \mathrm{NH}), 10.92(\mathrm{~s}, 1 \mathrm{H}$, $\mathrm{NH}) ;{ }^{13} \mathrm{C}-\mathrm{NMR}\left(\mathrm{DMSO}-d_{6}\right) \delta(\mathrm{ppm}): 17.3\left(\mathrm{NHCHCH}_{3}\right), 24.2\left(\mathrm{COCH}_{3}\right), 49.9\left(\mathrm{COOCH}_{3}\right), 52.1$ $\left(\mathrm{NHCHCH}_{3}\right), 120.0,122.4,124.4,126.4,128.9,129.0,131.6,134.21,139.4,142.2,162.3(\mathrm{CONH})$, $166.2(\mathrm{CONH}), 169.5(\mathrm{CONH}), 173.8\left(\mathrm{COOCH}_{3}\right), 190.0(\mathrm{COCO}) . \mathrm{IR}\left(\mathrm{cm}^{-1}\right): 3288,3124(\mathrm{NH}), 1741$, 1672, 1607, $1536(\mathrm{C}=\mathrm{O})$. Anal. Cacld for $\mathrm{C}_{21} \mathrm{H}_{21} \mathrm{~N}_{3} \mathrm{O}_{6}$ : C, 61.31; H, 5.14; N, 10.21. Found: C, 61.60; H, 5.34; N, 10.00 .

Methyl 2-(4-(2-(2-acetamidophenyl)-2-oxoacetamido)benzamido)-3-methylbutanoate (4b). White powder, mp: $178-180{ }^{\circ} \mathrm{C}$, yield $81 \% .{ }^{1} \mathrm{H}-\mathrm{NMR}\left(\mathrm{CDCl}_{3}\right) \delta(\mathrm{ppm}): 1.00\left(\mathrm{t}, J=8.60 \mathrm{~Hz}, 6 \mathrm{H}, \mathrm{CH}\left(\mathrm{CH}_{3}\right)_{2}\right), 1.59$ $\left(\mathrm{m}, 1 \mathrm{H}, \mathrm{CHCH}\left(\mathrm{CH}_{3}\right)_{2}\right), 2.27\left(\mathrm{~s}, 3 \mathrm{H}, \mathrm{COCH}_{3}\right), 3.78\left(\mathrm{~s}, 3 \mathrm{H}, \mathrm{COOCH}_{3}\right), 4.78(\mathrm{~m}, 1 \mathrm{H}, \mathrm{CHCHNH}), 6.61$ $(\mathrm{d}, J=8.08 \mathrm{~Hz}, 1 \mathrm{H}), 7.17(\mathrm{t}, J=8.08 \mathrm{~Hz}, 1 \mathrm{H}), 7.64(\mathrm{t}, J=8.08 \mathrm{~Hz}, 1 \mathrm{H}), 7.78(\mathrm{~d}, J=8.08 \mathrm{~Hz}, 1 \mathrm{H})$, $7.86(\mathrm{~d}, J=8.08 \mathrm{~Hz}, 2 \mathrm{H}), 8.50$ (d, $J=8.08 \mathrm{~Hz}, 1 \mathrm{H}) 8.64(\mathrm{~d}, J=8.08 \mathrm{~Hz}, 1 \mathrm{H}), 8.99$ (s, 1H, NH), 10.79

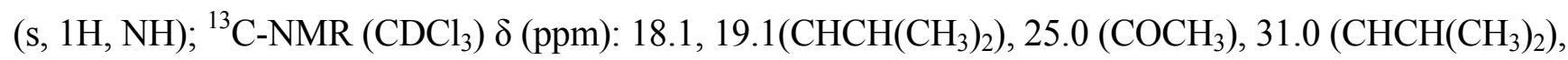
$52.4\left(\mathrm{COOCH}_{3}\right), 57.6\left(\mathrm{CHCH}\left(\mathrm{CH}_{3}\right)_{2}\right), 119.4,119.8,120.9,122.8,128.4,130.6,134.3,136.8,140.0$, 142.1,160.6 (CONH), $166.5(\mathrm{CONH}), 169.4(\mathrm{CONH}), 172.8\left(\mathrm{COOCH}_{3}\right), 190.8(\mathrm{COCO}) . \mathrm{IR}\left(\mathrm{cm}^{-1}\right)$ : 
$3293(\mathrm{NH}), 1747,1679,1634,1608,1526(\mathrm{C}=\mathrm{O})$. Anal. Cacld. for $\mathrm{C}_{23} \mathrm{H}_{25} \mathrm{~N}_{3} \mathrm{O6}_{3}$ : C, 62.86; H, 5.73; N, 9.56. Found: C, 63.02; H, 5.96; N, 9.81 .

Methyl 2-(4-(2-(2-acetamidophenyl)-2-oxoacetamido)benzamido)-3-phenylpropanoate (4c). White powder, mp: $168-170{ }^{\circ} \mathrm{C}$, yield $83 \% .{ }^{1} \mathrm{H}-\mathrm{NMR}\left(\mathrm{CDCl}_{3}\right) \delta(\mathrm{ppm}): 2.24\left(\mathrm{~s}, 3 \mathrm{H}, \mathrm{COCH}_{3}\right), 3.25(\mathrm{~m}, 2 \mathrm{H}$, $\left.\mathrm{CHCH}_{2} \mathrm{C}_{6} \mathrm{H}_{5}\right), 3.80\left(\mathrm{~s}, 3 \mathrm{H}, \mathrm{COOCH}_{3}\right), 5.08\left(\mathrm{~m}, 1 \mathrm{H}, \mathrm{CHCH}_{2} \mathrm{C}_{6} \mathrm{H}_{5}\right), 6.56(\mathrm{~d}, J=7.36 \mathrm{~Hz}, 1 \mathrm{H}), 7.12(\mathrm{~d}$, $J=6.6 \mathrm{~Hz}, 1 \mathrm{H}), 7.15-7.29\left(\mathrm{~m}, 5 \mathrm{H}, \mathrm{CHCH}_{2} \mathrm{C}_{6} \mathrm{H}_{5}\right), 7.65(\mathrm{t}, J=6.6 \mathrm{~Hz}, 1 \mathrm{H}), 7.76\left(\mathrm{~m}, 4 \mathrm{H}, \mathrm{NHC}_{6} \mathrm{H}_{4} \mathrm{CO}\right)$, $8.47(\mathrm{~d}, J=8.08 \mathrm{~Hz}, 1 \mathrm{H}), 8.63(\mathrm{~d}, J=8.08 \mathrm{~Hz}, 1 \mathrm{H}), 9.01(\mathrm{~s}, 1 \mathrm{H}, \mathrm{NH}), 10.79(\mathrm{~s}, 1 \mathrm{H}, \mathrm{NH}) ;{ }^{13} \mathrm{C}-\mathrm{NMR}$ $\left(\mathrm{CDCl}_{3}\right) \delta(\mathrm{ppm}): 25.0\left(\mathrm{COCH}_{3}\right), 38.0\left(\mathrm{CHCH}_{2} \mathrm{C}_{6} \mathrm{H}_{5}\right), 49.2\left(\mathrm{COOCH}_{3}\right), 52.6\left(\mathrm{CHCH}_{2} \mathrm{C}_{6} \mathrm{H}_{5}\right), 118.8$, $119.8,121.0,122.8,127.3,128.39,128.7,129.4,130.5,134.4,135.9,136.9,139.9,142.1,160.3$ $(\mathrm{CONH}), 166.0(\mathrm{CONH}), 169.4(\mathrm{CONH}), 172.1\left(\mathrm{COOCH}_{3}\right), 190.6(\mathrm{COCO}) . \mathrm{IR}\left(\mathrm{cm}^{-1}\right): 3250,3116$ $(\mathrm{NH}), 1750,1679,1635,1608,1523(\mathrm{C}=\mathrm{O})$. Anal. Calcd for $\mathrm{C}_{27} \mathrm{H}_{25} \mathrm{~N}_{3} \mathrm{O}_{6}$ : C, 66.52; H, 5.17; N, 8.62. Found: C, 66.67; H, 5.24; N, 8.88.

Methyl 2-(4-(2-(2-acetamidophenyl)-2-oxoacetamido)benzamido)-2-methylpropanoate (4d). White powder, mp: $212-218{ }^{\circ} \mathrm{C}$, yield 76\%. ${ }^{1} \mathrm{H}-\mathrm{NMR}\left(\mathrm{DMSO}-d_{6}\right) \delta(\mathrm{ppm}): 1.46\left(\mathrm{~s}, 6 \mathrm{H}, \mathrm{HNC}\left(\mathrm{CH}_{3}\right)_{2}\right), 1.99$ $\left(\mathrm{s}, 3 \mathrm{H}, \mathrm{COCH}_{3}\right), 3.58\left(\mathrm{~s}, 3 \mathrm{H}, \mathrm{COOCH}_{3}\right), 7.63-7.87\left(\mathrm{~m}, 8 \mathrm{H}, \mathrm{NHC}_{6} \mathrm{H}_{4} \mathrm{COCONHC}_{6} \mathrm{H}_{4} \mathrm{CO}\right), 8.54(\mathrm{~s}, 1 \mathrm{H}$, $\mathrm{NH}), 10.56(\mathrm{~s}, 1 \mathrm{H}, \mathrm{NH}), 10.90(\mathrm{~s}, 1 \mathrm{H}, \mathrm{NH}) ;{ }^{13} \mathrm{C}-\mathrm{NMR}\left(\mathrm{DMSO}-d_{6}\right) \delta(\mathrm{ppm}): 25.6\left(\mathrm{COCH}_{3}\right), 40.5$

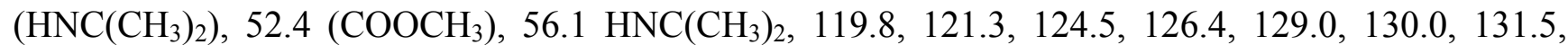
134.2, 138.1, 142.20,158.0 (CONH), $165.9(\mathrm{CONH}), 169.5(\mathrm{CONH}), 175.1\left(\mathrm{COOCH}_{3}\right), 189.5$ (COCO). IR $\left(\mathrm{cm}^{-1}\right): 3428(\mathrm{NH}), 1749,1680,1637,1525(\mathrm{C}=\mathrm{O})$. Anal. Calcd. for $\mathrm{C}_{22} \mathrm{H}_{23} \mathrm{~N}_{3} \mathrm{O}_{6}$ : C, 62.11; H, 5.45; N,9.88. Found: C, 62.38; H, 5.66; N, 10.07 .

Dimethyl 2-(4-(2-(2-acetamidophenyl)-2-oxoacetamido)benzamido)succinate (4e). White powder, mp: 154-156 ${ }^{\circ} \mathrm{C}$, yield 82\%. ${ }^{1} \mathrm{H}-\mathrm{NMR}$ (DMSO-d $) \delta(\mathrm{ppm}): 1.99\left(\mathrm{~s}, 3 \mathrm{H}, \mathrm{COCH}_{3}\right), 2.85-2.98(\mathrm{~m}, 2 \mathrm{H}$, $\left.\mathrm{CHCH}_{2} \mathrm{COOCH}_{3}\right), 3.62\left(\mathrm{~s}, 3 \mathrm{H}, \mathrm{COOCH}_{3}\right), 3.65\left(\mathrm{~s}, 3 \mathrm{H}, \mathrm{COOCH}_{3}\right), 4.83\left(\mathrm{~m}, 1 \mathrm{H}, \mathrm{HNCHCH}_{2}\right), 7.28-7.87$ $\left(\mathrm{m}, 8 \mathrm{H}, \mathrm{NHC}_{6} \mathrm{H}_{4} \mathrm{COCONH} \mathrm{C} \mathrm{H}_{4} \mathrm{CO}\right), 8.86(\mathrm{~d}, 1 \mathrm{H}, \mathrm{NH}), 10.54(\mathrm{~s}, 1 \mathrm{H}, \mathrm{NH}), 10.93$ (s, 1H, NH); ${ }^{13} \mathrm{C}-\mathrm{NMR}\left(\mathrm{DMSO}-d_{6}\right) \delta(\mathrm{ppm}): 23.9\left(\mathrm{COCH}_{3}\right), 36.0\left(\mathrm{NHCHCH}_{2} \mathrm{CO}\right), 49.8\left(\mathrm{NHCHCH}_{2} \mathrm{CO}\right), 52.3$ $\left(\mathrm{COOCH}_{3}\right), 52.8\left(\mathrm{COOCH}_{3}\right), 119.9,122.4,124.4,125.7,128.9,129.5,131.55,134.2,138.1,141.5$, $162.3(\mathrm{CONH}), 166.2(\mathrm{CONH}), 169.5(\mathrm{CONH}), 171.1\left(\mathrm{COOCH}_{3}\right), 171.9\left(\mathrm{COOCH}_{3}\right), 189.5(\mathrm{COCO})$. IR $\left(\mathrm{cm}^{-1}\right)$ : $3295(\mathrm{NH}), 1748,1667,1608,1526(\mathrm{C}=\mathrm{O})$. Anal. Calcd. for $\mathrm{C}_{27} \mathrm{H}_{25} \mathrm{~N}_{3} \mathrm{O}_{6}$ : C, 66.52; H, 5.17; N, 8.62. Found: C, 66.33; H, 5.26; N, 8.90.

Methyl 3-(4-(2-(2-acetamidophenyl)-2-oxoacetamido)benzamido)propanoate (4f). White powder, mp: 154-156 ${ }^{\circ} \mathrm{C}$, yield 88\%. ${ }^{1} \mathrm{H}-\mathrm{NMR}\left(\mathrm{DMSO}-d_{6}\right) \delta(\mathrm{ppm}): 1.99\left(\mathrm{~s}, 3 \mathrm{H}, \mathrm{COCH}_{3}\right), 2.60(\mathrm{t}, J=6.60 \mathrm{~Hz}, 2 \mathrm{H}$, $\mathrm{NHCH}_{2} \mathrm{CH}_{2} \mathrm{CO}$ ), 3.49 (q, 2H, $\mathrm{NHCH}_{2} \mathrm{CH}_{2} \mathrm{CO}$ ), 3.61 (s, 3H, $\left.\mathrm{COOCH}_{3}\right), 7.29-7.85$ (m, 8H, $\left.\mathrm{NHC}_{6} \mathrm{H}_{4} \mathrm{COCONH} \mathrm{C} \mathrm{H}_{4} \mathrm{CO}\right), 8.51(\mathrm{~s}, 1 \mathrm{H}, \mathrm{NH}), 10.55(\mathrm{~s}, 1 \mathrm{H}, \mathrm{NH}), 10.90(\mathrm{~s}, 1 \mathrm{H}, \mathrm{NH}) ;{ }^{13} \mathrm{C}-\mathrm{NMR}$ $\left(\mathrm{DMSO}-d_{6}\right) \delta$ (ppm): $23.9\left(\mathrm{COCH}_{3}\right), 34.1\left(\mathrm{NHCH}_{2} \mathrm{CH}_{2} \mathrm{CO}\right), 36.1\left(\mathrm{NHCH}_{2} \mathrm{CH}_{2} \mathrm{CO}\right), 52.0\left(\mathrm{COOCH}_{3}\right)$, 119.9, 122.4, 124.4, 125.6, 128.6, 130.4, 131.6, 134.3, 138.2, 141.1, 162.4 (CONH), $166.3(\mathrm{CONH})$, $169.5(\mathrm{CONH}), 172.4\left(\mathrm{COOCH}_{3}\right), 189.6(\mathrm{COCO}) . \mathrm{IR}\left(\mathrm{cm}^{-1}\right): 3295(\mathrm{NH}), 1742,1666,1635,1608$, $1527(\mathrm{C}=\mathrm{O})$. Anal. Calcd. for $\mathrm{C}_{21} \mathrm{H}_{21} \mathrm{~N}_{3} \mathrm{O}_{6}: \mathrm{C}, 61.31 ; \mathrm{H}, 5.14 ; \mathrm{N}, 10.21$. Found: $\mathrm{C}, 61.09 ; \mathrm{H}, 5.23 ; \mathrm{N}, 10.48$. 
Methyl 4-(4-(2-(2-acetamidophenyl)-2-oxoacetamido)benzamido)butanoate (4g). White powder, mp: $118-120{ }^{\circ} \mathrm{C}$, yield 86\%. ${ }^{1} \mathrm{H}-\mathrm{NMR}$ (DMSO- $\left.d_{6}\right) \delta(\mathrm{ppm}): 1.77\left(\mathrm{~m}, 2 \mathrm{H}, \mathrm{NHCH}_{2} \mathrm{CH}_{2} \mathrm{CH}_{2} \mathrm{CO}\right.$ ), $1.99(\mathrm{~s}, 3 \mathrm{H}$, $\left.\mathrm{COCH}_{3}\right), 2.37$ (t, $J=7.32 \mathrm{~Hz}, 2 \mathrm{H}, \mathrm{NHCH}_{2} \mathrm{CH}_{2} \mathrm{CH}_{2} \mathrm{CO}$ ), 3.27 (q, $2 \mathrm{H}, \mathrm{NHCH}_{2} \mathrm{CH}_{2} \mathrm{CH}_{2} \mathrm{CO}$ ), 3.58 $\left(\mathrm{s}, 3 \mathrm{H}, \mathrm{COOCH}_{3}\right), 7.28$ (t, $\left.J=7.3,2.2 \mathrm{~Hz}, 1 \mathrm{H}\right), 7.64-7.85$ (m, 7H, Ar), 8.42 (brs, 1H, NH), 10.55 $(\mathrm{s}, 1 \mathrm{H}, \mathrm{NH}), 10.89(\mathrm{~s}, 1 \mathrm{H}, \mathrm{NH}) ;{ }^{13} \mathrm{C}-\mathrm{NMR}\left(\mathrm{DMSO}-d_{6}\right) \delta(\mathrm{ppm}): 23.9\left(\mathrm{NHCH}_{2} \mathrm{CH}_{2} \mathrm{CH}_{2} \mathrm{CO}\right), 24.2$ $\left(\mathrm{COCH}_{3}\right), 31.4\left(\mathrm{NHCH}_{2} \mathrm{CH}_{2} \mathrm{CH}_{2} \mathrm{CO}\right), 41.6\left(\mathrm{NHCH}_{2} \mathrm{CH}_{2} \mathrm{CH}_{2} \mathrm{CO}\right), 51.8\left(\mathrm{COOCH}_{3}\right), 119.9,122.4$, 124.4, 125.6, 128.6, 130.7, 131.6, 134.3, 138.7, 141.0, $162.3(\mathrm{CONH}), 166.2(\mathrm{CONH}), 169.5(\mathrm{CONH})$, $173.7\left(\mathrm{COOCH}_{3}\right), 189.7$ (COCO). IR ( $\left.\mathrm{cm}^{-1}\right)$ : $3343(\mathrm{NH}), 1738,1661,1630,1600,1527(\mathrm{C}=\mathrm{O})$. Anal. Calcd. for $\mathrm{C}_{22} \mathrm{H}_{23} \mathrm{~N}_{3} \mathrm{O}_{6}$ : C, 62.11; H, 5.45; N, 9.88. Found: C, 61.37; H, 5.67; N, 10.04 .

Methyl 6-(4-(2-(2-acetamidophenyl)-2-oxoacetamido)benzamido)hexanoate (4h). White powder, mp: $\quad 180-182 \quad{ }^{\circ} \mathrm{C}$, yield $83 \% . \quad{ }^{1} \mathrm{H}-\mathrm{NMR} \quad\left(\mathrm{DMSO}-d_{6}\right) \quad \delta \quad(\mathrm{ppm}): \quad 1.32-1.36 \quad(\mathrm{~m}, \quad 2 \mathrm{H}$, $\mathrm{NHCH}_{2} \mathrm{CH}_{2} \mathrm{CH}_{2} \mathrm{CH}_{2} \mathrm{CH}_{2} \mathrm{CO}$ ), 1.49-1.56 (m, 4H, $\mathrm{NHCH}_{2} \mathrm{CH}_{2} \mathrm{CH}_{2} \mathrm{CH}_{2} \mathrm{CH}_{2} \mathrm{CO}$ ), 2.00 (s, 3H, $\mathrm{COCH}_{3}$ ), $2.31\left(\mathrm{t}, J=7.32 \mathrm{~Hz}, 2 \mathrm{H}, \mathrm{NHCH}_{2} \mathrm{CH}_{2} \mathrm{CH}_{2} \mathrm{CH}_{2} \mathrm{CH}_{2} \mathrm{CO}\right.$ ), 3.25 (t, $J=5.88 \mathrm{~Hz}, 2 \mathrm{H}, \mathrm{NHCH}_{2} \mathrm{CH}_{2} \mathrm{CH}_{2} \mathrm{CH}_{2}$ $\mathrm{CH}_{2} \mathrm{CO}$ ), $3.63\left(\mathrm{~s}, 3 \mathrm{H}, \mathrm{COOCH}_{3}\right), 7.28(\mathrm{t}, J=5.2 \mathrm{~Hz}, 1 \mathrm{H}), 7.64-7.85$ (m, 7H, Ar), 8.38 (brs, 1H, NH), $10.55(\mathrm{~s}, 1 \mathrm{H}, \mathrm{NH}), 10.88(\mathrm{~s}, 1 \mathrm{H}, \mathrm{NH}) ;{ }^{13} \mathrm{C}-\mathrm{NMR}$ (DMSO-d 6 ) $\delta(\mathrm{ppm}): 24.76\left(\mathrm{COCH}_{3}\right), 26.5$ $\left(\mathrm{NHCH}_{2} \mathrm{CH}_{2} \mathrm{CH}_{2} \mathrm{CH}_{2} \mathrm{CH}_{2} \mathrm{CO}\right), 29.4\left(\mathrm{NHCH}_{2} \mathrm{CH}_{2} \mathrm{CH}_{2} \mathrm{CH}_{2} \mathrm{CH}_{2} \mathrm{CO}, 33.8\left(\mathrm{NHCH}_{2} \mathrm{CH}_{2} \mathrm{CH}_{2} \mathrm{CH}_{2} \mathrm{CH}_{2} \mathrm{CO}\right)\right.$, $41.5\left(\mathrm{NHCH}_{2} \mathrm{CH}_{2} \mathrm{CH}_{2} \mathrm{CH}_{2} \mathrm{CH}_{2} \mathrm{CO}\right), 51.7\left(\mathrm{COOCH}_{3}\right), 119.9,122.4,124.4,126.5,128.5,130.8,131.6$, 134.3, 138.2, 140.9, $162.3(\mathrm{CONH}), 166.0(\mathrm{CONH}), 169.5(\mathrm{CONH}), 173.9\left(\mathrm{COOCH}_{3}\right), 189.7(\mathrm{COCO})$. IR $\left(\mathrm{cm}^{-1}\right)$ : $3290(\mathrm{NH}), 1738,1664,1637,1608,1528(\mathrm{C}=\mathrm{O})$. Anal. Calcd. for $\mathrm{C}_{24} \mathrm{H}_{27} \mathrm{~N}_{3} \mathrm{O}_{6}: \mathrm{C}, 63.56$; H, 6.00; N, 9.27. Found: C, 63.38; H, 6.13; N, 9.53.

Methyl 1-(4-(2-(2-acetamidophenyl)-2-oxoacetamido)benzamido)cyclopentanecarboxylate (4i). White powder, mp: $238-240{ }^{\circ} \mathrm{C}$, yield 78\%. ${ }^{1} \mathrm{H}-\mathrm{NMR}$ (DMSO- $\left.d_{6}\right) \delta(\mathrm{ppm}): 1.69-1.71 \quad(\mathrm{~m}, 4 \mathrm{H}$, $\left.\mathrm{CH}_{2} \mathrm{CH}_{2} \mathrm{CH}_{2} \mathrm{CH}_{2}\right), 1.99$ (s, 3H, $\left.\mathrm{COCH}_{3}\right), 2.00-2.13$ (m, 4H, $\left.\mathrm{CH}_{2} \mathrm{CH}_{2} \mathrm{CH}_{2} \mathrm{CH}_{2}\right), 3.58\left(\mathrm{~s}, 3 \mathrm{H}, \mathrm{COOCH}_{3}\right)$, $7.28(\mathrm{t}, J=6.16 \mathrm{~Hz}, 1 \mathrm{H}$ ), $7.63-7.87$ (m, 7H, Ar), 8.50(s, 1H, NH), 10.55(s, 1H, NH), $10.90(\mathrm{~s}, 1 \mathrm{H}, \mathrm{NH})$; ${ }^{13} \mathrm{C}-\mathrm{NMR}\left(\mathrm{DMSO}-d_{6}\right) \delta(\mathrm{ppm}): 24.1\left(\mathrm{CH}_{2} \mathrm{CH}_{2} \mathrm{CH}_{2} \mathrm{CH}_{2}\right), 24.7\left(\mathrm{COCH}_{3}\right), 39.8\left(\mathrm{CH}_{2} \mathrm{CH}_{2} \mathrm{CH}_{2} \mathrm{CH}_{2}\right), 52.5$ $\left(\mathrm{COOCH}_{3}\right), 66.2(\mathrm{C}), 119.8,122.5,124.5,125.9,129.0,130.0,131.5,134.2,138.0,141.3,162.2$ $(\mathrm{CONH}), 166.4(\mathrm{CONH}), 169.5(\mathrm{CONH}), 174.9\left(\mathrm{COOCH}_{3}\right), 189.5(\mathrm{COCO}) . \mathrm{IR}\left(\mathrm{cm}^{-1}\right): 3291,3114$ $(\mathrm{NH}), 1746,1679,1633,1607,1524(\mathrm{C}=\mathrm{O})$. Anal. Calcd. for $\mathrm{C}_{24} \mathrm{H}_{25} \mathrm{~N}_{3} \mathrm{O}_{6}$ (451.47): C, 63.85; H, 5.58; N, 9.31. Found: C, 64.06; H, 5.65; N, 9.04.

\section{Conclusions}

In conclusion, we have demonstrated that reaction of $\mathrm{N}$-acetylisatin (1) with 4-aminobenzoic acid (2), either using conventional heating or microwave irradiation affords the $\alpha$-ketoamide 4-(2-(2-acetamidophenyl)-2-oxoacetamido)benzoic acid (3) in good yield. All the spectral data proved the ring opening structure in which the 4-aminobenzoic acid attacks $\mathrm{C} 2$ rather than $\mathrm{C} 3$. Reaction of the 4-aminobenzoic acid derivative 3 with different amino acid esters using Oxyma/DIC afforded a novel series of 4-[2-(2-acetylaminophenyl)-2-oxo-acetylamino]benzoyl amino acid esters 4a-i. OxymaPure/DIC showed clear superiority to HOBt/DIC and carbodiimide alone in terms of yield and purity. 


\section{Supplementary Materials}

Supplementary materials can be accessed at: http:/www.mdpi.com/1420-3049/18/12/14747/s1.

\section{Acknowledgments}

The authors thank the Deanship of Scientific Research at King Saud University for funding this work through research group "RGP-VPP-234". The work in Barcelona was partially supported by CICYT (CTQ2012-30930) and the Generalitat de Catalunya (2009SGR 1024).

\section{Conflicts of interest}

The authors declare no conflict of interest.

\section{References}

1. Li, Z.; Ortega-Vilain, A.C.; Patil, G.S.; Chu, D.L.; Foreman, J.E.; Eveleth, D.D.; Powers, J.C. Novel peptidyl $\alpha$-Keto amide inhibitors of calpains and other cysteine proteases. J. Med. Chem. 1996, 39, 4089-4098.

2. James, D.A.; Koya, K.; Li, H.; Liang, G.; Xia, Z.; Ying, W.; Wu, Y.; Sun, L. Indole- and indolizine-glyoxylamides displaying cytotoxicity against multidrug resistant cancer cell lines. Bioorg. Med. Chem. Lett. 2008, 18, 1784-1787.

3. Montalban, A.G.; Boman, E.; Chang, C.D.; Ceide, S.C.; Dahl, R.; Dalesandro, D.; Delaet, N.G.J.; Erb, E.; Ernst, J.T.; Gibbs, A.; et al. The design and synthesis of novel $\alpha$-ketoamide-based p38 MAP kinase inhibitors. J. Bioorg. Med. Chem. Lett. 2008, 18, 1772-1777.

4. Yu, P.-F.; Chen, H.; Wang, J.; He, C.-X.; Cao, B.; Li, M.; Yang, N.; Lei, Z.Y.; Cheng, M.-S. Design, synthesis and cytotoxicity of novel podophyllotoxin derivatives. Chem. Pharm. Bull. 2008, 56, 831-834.

5. Perni, R.B.; Farmer, L.J.; Cottrell, K.M.; Court, J.J.; Courtney, L.F.; Deininger, D.D.; Gates, C.A.; Harbeson, S.L.; Kim, J.L.; Lin, C.; et al. Inhibitors of hepatitis C virus NS3.4A protease. Part 3: P2 proline variants. Bioorg. Med. Chem. Lett. 2004, 14, 1939-1942.

6. Victor, F.; Lamar, J.; Snyder, N.; Yip, Y.; Guo, D.; Yumibe, N.; Johnson, R.B.; Wang, Q.M.; Glass, J.I.; Chen, S.H. P1 and P3 optimization of novel bicycloproline P2 bearing tetrapeptidyl $\alpha$-ketoamide based HCV protease inhibitors. Bioorg. Med. Chem. Lett. 2004, 14, 257-261.

7. Banfi, L.; Guanti, G.; Riva, R. Passerini multicomponent reaction of protected alpha-aminoaldehydes as a tool for combinatorial synthesis of enzyme inhibitors. J. Chem. Soc. Chem. Commun. 2000, 985-986.

8. Nakamura, M.; Inoue, J.; Yamada, T. A two-step, one-pot synthesis of diverse $N$-pyruvoyl amino acid derivatives using the Ugi reaction. Bioorg. Med. Chem. Lett. 2000, 10, 2807-2810.

9. Xu, P.; Lin, W.; Zhou, X. Synthesis of a peptidomimetic HCMV protease inhibitor library. Synthesis 2002, 8, 1017-1026.

10. Chen, J.J.; Deshpande, V. Rapid synthesis of $\alpha$-ketoamides using microwave irradiation simultaneous cooling method. Tetrahedron Lett. 2003, 44, 8873-8876. 
11. Faggi, C.; Neo, A.G.; Marcaccini, S.; Menchi, G.; Revuelta, J. Ugi four-component condensation with two cleavable components: The easiest synthesis of 2, $\mathrm{N}$-diarylglycines. Tetrahedron Lett. 2008, 49, 2099-2102.

12. Grassot, J.M.; Masson, G.; Zhu, J. Synthesis of $\alpha$-ketoamides by a molecular-sieves-promoted formal oxidative coupling of aliphatic aldehydes with isocyanides. Angew. Chem. Int. Ed. 2008, 47, 947-950.

13. Chen, Y.H.; Zhang, Y.H.; Zhang, H.J.; Liu, D.Z.; Gu, M.; Li, J.Y.; Wu, F.; Zhu, X.Z.; Li, J.; Nan, F.J. Design, synthesis, and biological evaluation of isoquinoline-1,3,4-trione derivatives as potent Caspase-3 inhibitors. J. Med. Chem. 2006, 49, 1613-1623.

14. Song, B.; Wang, S.; Sun, C.; Deng, H.; Xu, B. Cesium carbonate promoted aerobic oxidation of arylacetamides: An efficient access to N-substituted $\alpha$-keto amides. Tetrahedon Lett. 2007, 48, 8982-8986.

15. Chen, C.T.; Bettigeri, S.; Weng, S.S.; Pawar, V.D.; Lin, Y.H.; Liu, C.Y.; Lee, W.Z. Asymmetric aerobic oxidation of $\alpha$-hydroxy acid derivatives by $C_{4}$-Symmetric, Vanadate-Centered, Tetrakisvanadyl(V) clusters derived from $N$-Salicylidene- $\alpha$-aminocarboxylates. J. Org. Chem. 2007, 72, 8175-8185.

16. Lamberth, C.; Jeanguenat, A.; Cederbaum, F.; de Mesmaecker, A.; Zeller, M.; Kempf, H.J.; Zeun, R. Multicomponent reactions in fungicide research: The discovery of mandipropamid. Bioorg. Med. Chem. 2008, 16, 1531-1545.

17. Sanz, R.; Castroviejo, M.P.; Guilarte, V.; Pérez, A.; Fañanás, F.J. Regioselective synthesis of 4- and 7-Alkoxyindoles from 2,3-Dihalophenols: Application to the preparation of indole inhibitors of phospholipase A2. J. Org. Chem. 2007, 72, 5113-5118.

18. Maresh, J.J.; Giddings, L.-A.; Friedrich, A.; Loris, E.A.; Panjikar, S.; Trout, B.L.; Stöckigt, J.; Peters, B.; O’Connor, S.E. Strictosidine synthase: Mechanism of a pictet-spengler catalyzing enzyme. J. Am. Chem. Soc. 2008, 130, 710-723.

19. Hua, R.; Takeda, H.A.; Abe, Y.; Tanaka, M. Reactions of a carbamoylstannane with acid chlorides: Highly efficient synthesis of $\alpha$-Oxo amides. J. Org. Chem. 2004, 69, 974-976.

20. Arasappan, A.; Venkatraman, S.; Padilla, A.I.; Wu, W.; Meng, T.; Jin, Y.; Wong, J.; Prongay, A.; Girijavallabhan, V.; Njoroge, F.G. Practical and efficient method for amino acid derivatives containing $\beta$-quaternary center: Application toward synthesis of hepatitis C virus NS3 serine protease inhibitors. Tetrahedron Lett. 2007, 48, 6343-6347.

21. Zhang, L.; Sun, F.; Li, Y.; Sun, X.; Liu, X.; Huang, Y.; Zhang, L.-H.; Ye, X.-S.; Xiao, J. Rapid synthesis of iminosugar derivatives for cell-based in situ screening: Discovery of "Hit" compounds with anticancer activity. ChemMedChem 2007, 2, 1594-1597.

22. González, J.F.; de la Cuesta, E.; Avendaño, C. Atom-efficient synthesis of 2,6-diazacyclophane compounds through alcoholysis/reduction of 3-nitroarylmethylene-2,5-piperazinediones. Tetrahedron 2008, 64, 2762-2771.

23. Popp, F.D.; Piccirilli, M. The reaction of N-acetylisatin with amines. J. Heterocycl. Chem. 1971, $8,473-475$.

24. Obafemi, C.A.; Taiwo, F.O.; Iwalewai, E.O.; Akinpelu, D.A. Synthesis, antibacterial and anti-inflammatory activities of some 2-phenylglyoxylic acid derivatives. Int. J. Life Sci. 2013, 2 , $22-36$. 
25. Andreani, A.; Burnelli, S.; Granaiola, M.; Leoni, A.; Locatelli, A.; Morigi, R.; Rambaldi, M.; Varoli, L.; Cremonini, M.A.; Placucci, G.; et al. New isatin derivatives with antioxidant activity. Eur. J. Med. Chem. 2010, 45, 1374-1378.

26. Boechat, N.; Kover, W.B.; Bastos, M.M.; Pinto, A.C.; Maciel, L.C.; Mayer, L.M.U.; da Silva, F.S.Q.; Sá, P.M.; Mendonça, J.S.; Wardella, S.M.S.V.; et al. N-Acyl-3,3-difluoro-2-oxoindoles as versatile intermediates for the preparation of different 2,2-difluorophenylacetic derivatives. J. Braz. Chem. Soc. 2008, 19, 445-457.

27. Ghazzali, M.; El-Faham, A.; Abdel-Megeed, A.; Al-Farhan, K. Microwave-assisted synthesis, structural elucidation and biological assessment of 2-(2-acetamidophenyl)-2-oxo- $N$ phenyl acetamide and $\mathrm{N}$-(2-(2-oxo-2(phenylamino)acetyl)phenyl)propionamide derivatives. J. Mol. Struct. 2012, 1013, 163-167.

28. Cheah, W.C.; Black, D.S.; Goh, W.K.; Kumar, N. Synthesis of anti-bacterial peptidomimetics derived from N-acylisatins. Tetrahedron Lett. 2008, 49, 2965-2968.

29. Cheah, W.C.; Wood, K.; Black, D.S.; Kumar, N. Facile ring-opening of $N$-acylisatins for the development of novel peptidomimetics. Tetrahedron 2011, 67, 7603-7610.

30. Hossain, M.M.; Islam, R.M.; Saha, S.K.; Islam, M.K. An efficient microwave-assisted synthesis of dihydropyrazinones and bis-benzoylketones. Tetrahedron Lett. 2010, 51, 1155-1157.

31. Somogy, L. Transformation of Isatin 3-Acylhydrazones under acetylating conditions: Synthesis and structure elucidation of 1,5'-Disubstituted 3'-Acetylspiro[oxindole-3,2'-[1,3,4]oxadiazolines]. Bull. Chem. Soc. Jpn. 2001, 74, 873-881.

32. Pandey, S.K. Synthesis and evaluation of anti-inflammatory activity of 3-substituted indole derivatives. J. Pharm. Res. 2010, 3, 2738-2741.

33. Verma, M.; Pandeya, S.N.; Singh, K.N.; Stables, J. Anticonvulsant activity of Schiff bases of isatin derivatives. Acta Pharm. 2004, 54, 49-56.

34. Subirós-Funosas, R.; Prohens, R.; Barbas, R.; El-Faham, A.; Albericio, F. Oxyma: An efficient additive for peptide synthesis to replace benzotriazole-based HOBt and HOAt with a lower risk of explosion. Chem. Eur. J. 2009, 15, 9394-9403.

35. Subirós-Funosas, R.; Khattab, S.N.; Nieto-Rodriguez, L.; El-Faham, A.; Albericio, F. Oxyma as cyanooxime building block for the construction of versatile reagents assisting acylation reactions in peptide and organic chemistry. Aldrichimica Acta 2013, 40, 21-40.

36. Jad, Y.E.; Khattab, S.N.; El-Faham, A.; Albericio, F. Oxime-based carbonates as useful reagents for both N-protection and peptide coupling. Molecules 2012, 17, 14361-14376.

37. Khattab, S.N.; Subirós-Funosas, R.; El-Faham, A.; Albericio, F. Screening of $N$-alkyl-cyanoacetamido oximes as substitutes for $N$-Hydroxysuccinimide. ChemistryOpen 2012, 1, 147-152.

38. Subiros-Funosas, R.; El-Faham, A.; Albericio, F. Use of Oxyma as pH modulatory agent to be used in the prevention of base-driven side reactions and its effect on 2-chlorotrityl chloride resin. Biopolymer 2012, 98, 89-97.

39. El-Faham, A.; Albericio, F. Peptide coupling reagents, more than a letter soup. Chem. Rev. 2011, $111,6557-6602$.

40. Subiros-Funosas, R.; El-Faham, A.; Albericio, F. Aspartimide formation in peptide chemistry: Occurrence, prevention strategies and the role of $N$-hydroxylamines. Tetrahedron 2011, 67, 8595-8606. 
41. El-Faham, A.; Subiros-Funosas, R.; Albericio, F. A novel family of onium salts based upon isonitroso Meldrum's acid proves useful as peptide coupling reagents. Eur. J. Org. Chem. 2010, 2010, 3641-3649.

42. Subiros-Funosas, R.; Acosta, G.A.; El-Faham, A.; Albericio, F. Microwave irradiation and COMU: A superior tool for solid phase peptide synthesis. Tetrahedron Lett. 2009, 50, 6200-6202.

43. Kudelko, A.; Zieliński, W. An efficient synthesis of new 2-aminomethyl-1,3,4-oxadiazoles from enantiomeric phenylglycine hydrazides. Tetrahedron 2009, 65, 1200-1206.

44. Li, J.; Sha, Y. A convenient synthesis of amino acid methyl esters. Molecules 2008, 13, 1111-1119.

Sample Availability: Samples of compounds are available from authors.

(C) 2013 by the authors; licensee MDPI, Basel, Switzerland. This article is an open access article distributed under the terms and conditions of the Creative Commons Attribution license (http://creativecommons.org/licenses/by/3.0/). 